\title{
Characterization of Biosurfactant-Containing Liposomes and Their Efficiency for Gene Transfection
}

\author{
Yoshinobu Ueno, ${ }^{a}$ Naohide Hirashima, ${ }^{a}$ Yoshikazu InoH,${ }^{a, b}$ Tadahide Furuno,,${ }^{a, b}$ and \\ Mamoru NAKANISHI $*, a, b$ \\ ${ }^{a}$ Graduate School of Pharmaceutical Sciences, Nagoya City University; 3-1 Tanabe-dori, Mizuho-ku, Nagoya 467-8603, \\ Japan: and ${ }^{b}$ School of Pharmacy, Aichi Gakuin University; 1-100 Kushumoto-cho, Chikusa-ku, Nagoya 464-8650, Japan. \\ Received September 11, 2006; accepted October 16, 2006; published online October 19, 2006
}

\begin{abstract}
Recently we showed significance of biosurfactants in the field of non-viral vectors for gene transfection. There, a biosurfactant, mannosylerythritol lipid A (MEL-A), especially increased the efficiency of gene transfection mediated with cationic liposomes. However, the molecular mechanism has not been well-understood yet. Here, through the examination of the ability of cationic liposomes containing an MEL (MEL-A, MEL-B or MEL-C) for important transfectional processes of the DNA capsulation and the membrane fusion with anionic liposomes, we found that MEL-A-containing liposomes increased both processes, but that MEL-B and MEL-Ccontaining liposomes just increased either of them. The results indicated that these kinds of the physicochemical properties in MEL-A-containing liposomes are able to increase the efficiency of liposome-mediated gene transfection.
\end{abstract}

Key words biosurfactant; cationic liposome; cationic cholesterol; gene transfection; membrane fusion

The establishment of an efficient and safe method for introducing exogenous nucleotides into mammalian cells is critical for basic sciences and clinical applications such as gene therapy. Among various methods for gene transfection, lipofection using cationic liposomes is considered to be a promising way to deliver foreign gene to the target cells without side-effects. $^{1-4)}$ Although several kinds of cationic liposomes for lipofection have been developed, further studies are still required to develop a non-viral vector which has comparable efficiency to viral vectors. We have been developing some new techniques and methodologies for the liposome-based gene transfection. ${ }^{5-10)}$ Recently, we introduced biosurfactants in this field. Biosurfactants are surface-active compounds which microorganisms growing on water-insoluble substrates produce. Biosurfactants, mannosylerythritol lipids (MELs), are abundantly produced by yeast Candida antarctica T-34 and have the advantages of low-toxicity and biodegradability. ${ }^{11)}$ When we previously compared the efficiency of gene transfection mediated by cationic liposomes with three different derivatives of MELs (MEL-A, MEL-B, or MEL-C), the transfection efficiencies are quite different among three kinds of MELs. That is, MEL-A di-acetylated at $4^{\prime}-O$ and $6^{\prime}-O$ of mannopyranosyl ring induced remarkable transfection activity using cationic liposomes, but neither MEL-B mono-acetylated at $6^{\prime}-O$ nor MEL-C mono-acetylated at $4^{\prime}-O$ of mannopyranosyl ring induced it. ${ }^{12,13)}$ It seemed that the solution in this problem will bring breakthrough in non-viral vectors for gene therapy.

In this study, we examined a couple of physicochemical properties of MEL-containing liposomes to clear up why MEL-A-containing liposomes could accelerate gene transfection. We found that MEL-A-containing liposomes exhibited the high activity in DNA capsulation and membrane fusion with anionic liposomes which are important properties for gene transfection. On the other hand, MEL-B and MELC-containing liposomes only increased either the capsulation or the membrane fusion. The present experimental results gave us an interesting explanation why MEL-A enhanced gene-transfection mediated with cationic liposomes.

\section{MATERIALS AND METHODS}

Materials The synthesis of a cationic cholesterol derivative, cholesteryl-3 $\beta$-carboxyamido ethylene- $N$-hydroxyethylamine (OH-Chol) was described in our previous paper. ${ }^{5)} 1,2-$ Dioleoyl-sn-glycero-3-phosphatidylethanolamine (DOPE) was obtained from Sigma (St. Louis, MO, U.S.A.). Luciferase plasmid (pRL-CMV) was obtained from Promega (Madison, WI, U.S.A.). NBD- and rhodamine-labeled PE were purchased from Molecular Probes (Eugene, OR, U.S.A. ${ }^{14)}$ Ethidium bromide was purchased from Wako Pure Chemical Industries (Osaka, Japan).

Biosurfactant MELs MEL-producing yeast (Candida antarctica T-34) was isolated from the exudate of a tree on Mt. Tsukuba in Japan. ${ }^{11)}$ MEL-A (4-O-[(4', $6^{\prime}$-di- $O$-acetyl$2^{\prime}, 3^{\prime}$-di- $O$-alkanoyl)- $\beta$-D-mannopyranosyl] meso-erythritol) was synthesized by growing the yeast on a fermentation medium consisted of $8 \%(\mathrm{v} / \mathrm{v})$ soybean oil, $0.2 \% \mathrm{NaNO}_{3}$, $0.02 \% \mathrm{KH}_{2} \mathrm{PO}_{4}, 0.02 \% \mathrm{MgSO}_{4}\left(7 \mathrm{H}_{2} \mathrm{O}\right), 0.1 \%$ yeast extract and tap water. MEL-A was extracted with ethyl acetate from the reaction medium. The crude MEL-A was purified by silica-gel (Wako-gel C-200) column chromatography with chloroform-acetate $(9: 1$ to $5: 5)$ mixture as solvent systems. MEL-B (4- $O$-[(6'-O-acetyl-2', $3^{\prime}$-di- $O$-alkanoyl)- $\beta$-D-mannopyranosyl $]$ meso-erythritol) and MEL-C (4-O-[(4'-O-acetyl$2^{\prime}, 3^{\prime}$-di- $O$-alkanoyl)- $\beta$-D-mannopyranosyl] meso-erythritol) were also prepared by the method described previously. ${ }^{11,13)}$ The molecular structures of MELs were shown in Fig. 1.

Preparation of Liposomes DOPE was combined with $\mathrm{OH}-\mathrm{Chol}$ in chloroform and dried with $\mathrm{N}_{2}$ gas to remove chloroform solvent. Liposomes were composed of $20 \mathrm{nmol}$ DOPE and $30 \mathrm{nmol} \mathrm{OH}-\mathrm{Chol}$. Each MEL was contained at a concentration of $10 \mathrm{nmol}$ in the liposomes (DOPE $+\mathrm{OH}-$ Chol) because it showed the maximum activity for gene transfection at this concentration. Mixtures were dried under reduced pressure to remove chloroform solvent. The dried 
(a) MEL-A

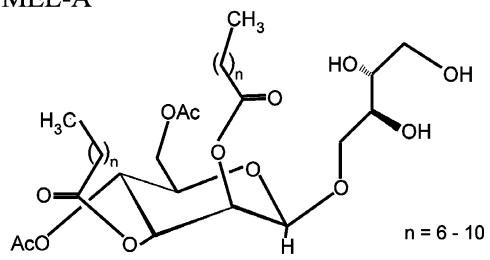

(b) MEL-B

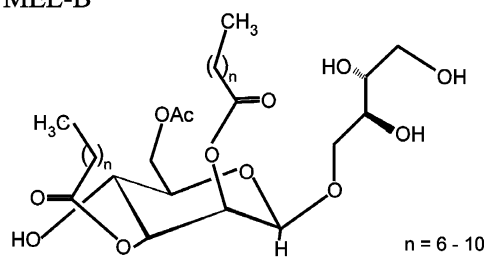

(c) MEL-C

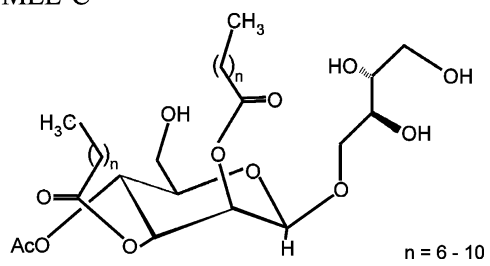

Fig. 1. Molecular Structures of Biosurfactant MELs Used in the Present Experiments

(a) Chemical structure of biosurfactant MEL-A. MEL-A consists of 4-O-(di-Oacetyl-di- $O$-alkanoyl- $\beta$-D-mannopyranosyl)-erythritol esterified two fatty acids and two acetic acids. (b, c) Chemical structure of MEL-B and MEL-C. These MELs consists of 4- $O$-(mono- $O$-acetyl-di- $O$-alkanoyl- $\beta$-D-mannopyranosyl)-erythritol esterified two fatty acids and an acetic acid.

film was vacuum desiccated for at least $30 \mathrm{~min}$ and suspended by vortexing and the samples were sonicated in a bath type sonicator (Branson model B 1200) to generate small unilamellar vesicles (SUVs) following our previous procedures. $^{5,12,13)}$

Ethidium Intercalation Assay The interaction between liposomes and DNA was examined by ethidium intercalation assay. When ethidium ions were added to a solution of DNA, they intercalate between the base pairs of DNA double helix, emitting an intense fluorescence. Interaction between liposomes and DNA was estimated relative decrease in the fluorescence intensity due to the interaction. Ethidium was excited at $520 \mathrm{~nm}$ and fluorescent intensity at $595 \mathrm{~nm}$ was observed. ${ }^{16,17)}$ The amount of DNA which is protected from intercalation of ethidium was calculated from the relative intensity to the maximum intensity obtained when ethidium was added to free plasmid DNA without liposomes.

Membrane Fusion Assay To investigate the effects of MELs in cationic liposomes on the membrane fusion, cationic liposomes with or without MELs were fused with anionic liposomes (DOPG: DOPE : DOPC $=1: 2: 1$ ). For the evaluation of membrane fusion, anionic liposomes containing $0.5 \%$ (mol) NBD-PE and $0.5 \%$ (mol) rhodamine-PE were mixed with cationic liposomes. Samples were excited at $488 \mathrm{~nm}$ and fluorescence spectra were obtained with a spectrofluorometer (RF5300PC; Shimadzu, Japan). Membrane fusion causes to decrease the efficiency of fluorescence resonance energy transfer (FRET) from NBD to rhodamine, resulting in increase of fluorescent intensity of NBD and decrease in that of rhodamine. ${ }^{18-20)}$ To show the time-course of (a)

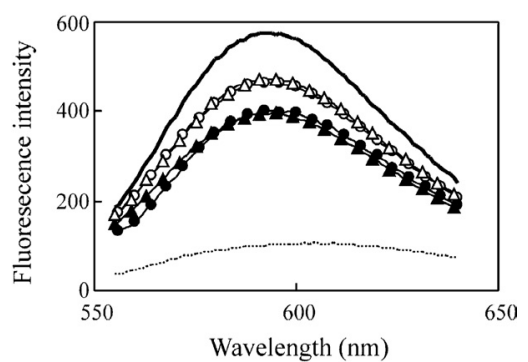

(b)

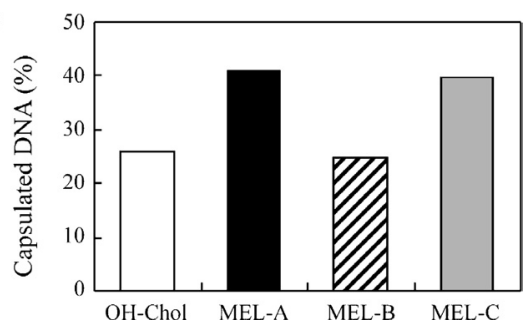

Fig. 2. The Interaction of DNA with Cationic Liposomes Containing MELs Monitored by EtBr Intercalation Assay

(a) Fluorescent spectra of EtBr intercalating to DNA. The fluorescence spectra of $\mathrm{EtBr}$ in the absence (a broken line) and the presence (a solid line) of DNA without cationic liposomes were shown. The spectra of $\mathrm{EtBr}$ in the presence of complexes composed of DNA and cationic liposomes without MELs (open circles) and with MEL-A (closed circles), MEL-B (open triangles), and MEL-C (closed triangles). (b) The capsulation levels of DNA was compared among cationic liposomes with MELs (MEL-A $-\mathrm{B}$, or $-\mathrm{C})$ and without MELs (OH-Chol).

membrane fusion between cationic and anionic liposomes, the NBD fluorescence intensity at $525 \mathrm{~nm}$ was plotted. Experiments were carried out in a sodium citrate/phosphate buffer solution $\left(\mathrm{pH} \mathrm{7.0)}\right.$ ) at $37^{\circ} \mathrm{C}$.

\section{RESULTS}

We first examined the intercalation of ethidium to DNA to measure the capsulation of DNA with cationic liposomes. Figure 2a shows the fluorescence spectra of ethidium with free DNA, with capsulated DNA, and without DNA. Fluoresecence intensity of ethidium was weakest in solution without DNA (a dotted line) and it became strongest in the solution of free DNA (a continuous line). The fluorescence intensity of ethidium depends on the DNA intercalation, and the fluorescence spectra in Fig. 2a show how much the DNA was capsulated for each cationic liposome containing MEL-A, MEL-B, or MEL-C. ${ }^{16,17)}$ As shown in Fig. 2a, DNAs were well capsulated with MEL-A- and MEL-C-containing liposomes (closed circles and closed triangles), but not well capsulated with MEL-B-containing liposomes (open triangles). The latter was almost similar to the value by the liposomes without biosurfactants (open circles). The efficiency of DNA capsulation by individual liposomes calculated from fluorescence intensity of ethidium showed that MEL-A-containing liposomes capsulated DNAs much more than MEL-B-containing liposomes and the liposomes without biosurfactants, as shown in Fig. 2b. The capsulation of DNA is considered to be caused by electrostatic interaction between cationic liposomes and DNA (phosphate polymers), and it facilitates the incorporation of DNAs to target cells. From the standpoint of the capsulation, MEL-C-containing liposomes seemed to capsulate DNAs as similar as MEL-A-containing liposomes. 
(a)

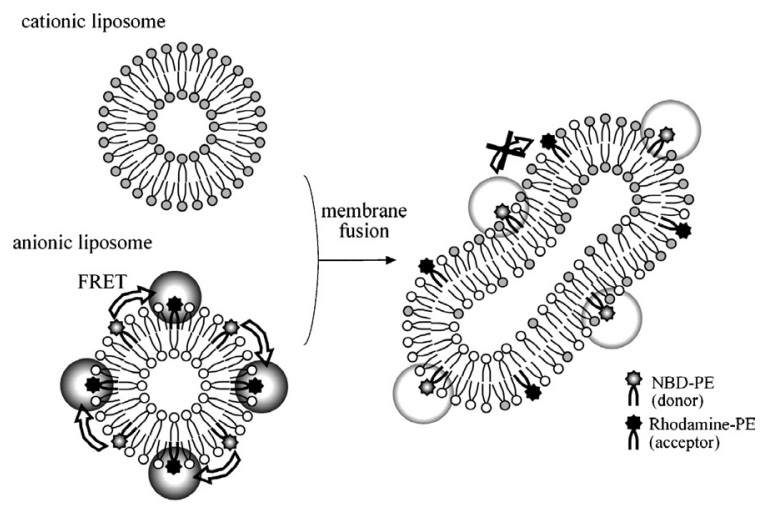

(b)

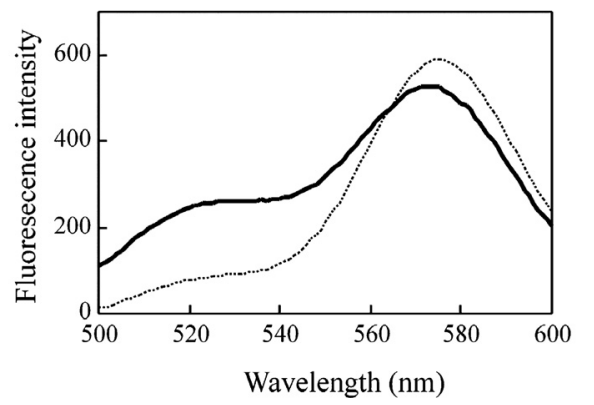

Fig. 3. The Decrease in FRET Efficiency from NBD-PE to RhodaminePE by Membrane Fusion

(a) The schematic representation of decrease in FRET efficiency from NBD-PE to rhodamine-PE by membrane fusion. (b) Fluorescent spectra of NBD and rhodamine in anionic liposomes before (a broken line) and at $10 \mathrm{~min}$ after the addition of cationic liposomes containing MEL-A (a solid line).

However, MEL-A significantly increased the gene transfection efficiency, while MEL-B and MEL-C hardly affected the transfection efficiency in our previous experiments. ${ }^{13)}$ These suggested that at least one more process was related to the high efficiency of gene transfection mediated by MEL-Acontaining liposomes.

Next, we studied the effects of MELs on the membrane fusion between cationic liposomes and anionic liposomes by fluorescence spectroscopy. The procedure is shown schematically in Fig. 3a. The membrane fusion was measured with the decrease in FRET efficiency between two fluorophores (NBD-PE (donor) and rhodamine-PE (acceptor)) in anionic liposomes according to the increase of their distance by mixing with non-fluoresecent lipids in cationic liposomes. Therefore, when the membrane fusion occurred between anionic liposomes and cationic liposomes, NBD fluorescence intensity increased at $c a .525 \mathrm{~nm}$ and rhodamine fluorescence at ca. $575 \mathrm{~nm}$ decreased, as shown in Fig. 3b. Figure 4a shows the time course of fluorescence intensity changes of NBD at $525 \mathrm{~nm}$ in anionic liposomes after the addition of cationic liposomes with or without MEL-A. The plateau level of fluorescence intensity after the addition of liposomes with MEL-A (after 10-15 min) increased to about three times as much as that in the case without biosurfactants, as shown in Fig. 4b. These results are well consistent with our previous findings that MEL-A-containing cationic liposomes facilitated the fusion with the plasma membrane of the target cells. ${ }^{13)}$ The data also indicated that MEL-A in cationic lipo- (a)

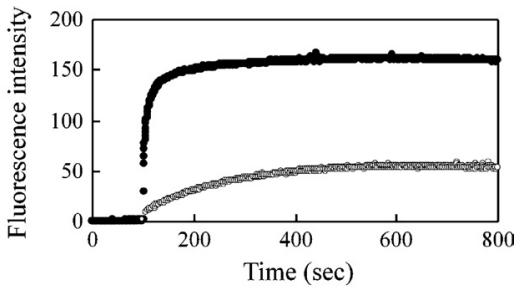

(b)

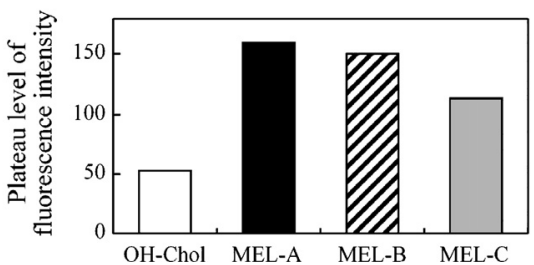

Fig. 4. The Membrane Fusion between MEL-containing Cationic Liposomes and Anionic Liposomes

(a) The time course of membrane fusion between cationic and anionic liposomes by FRET assay. The cationic liposomes with MEL-A (closed circles) or without MELs (open circles) were added to anionic liposomes containing NBD-PE and rhodamine-PE at $100 \mathrm{~s}$. The increase in fluorescence intensity of NBD according to the decrease in FRET efficiency by membrane fusion was monitored. (b) The plateau levels of the NBD fluorescence intensity were compared among cationic liposomes with MELs (MEL-A, -B, or -C) and without MELs (OH-Chol)

somes induced the membrane fusion with anionic liposomes more efficiently than MEL-C. MEL-B-containing liposomes showed the efficiency for the membrane fusion with anionic liposomes as similar as that of MEL-A-containing liposomes, however, they could hardly capsulate DNA as mentioned above (see Fig. 2).

\section{DISCUSSION}

We previously found that the sequential three processes were involved in gene transfection mediated by cationic liposomes. The first process is the attachment of liposome-DNA complexes to the plasma membrane of target cells, the second is the release of DNA from complexes through the membrane fusion, and the third is the delivery of DNA into the nucleus of target cells. ${ }^{10)} \mathrm{We}$ also showed that the biosurfactant MEL-A accelerated dramatically the efficiency of gene transfection but not other derivetives MEL-B and MEL-C. ${ }^{13)}$ Here we have analyzed the difference of physicochemical properties to affect the efficiency of gene transfection among liposomes containing MEL-A, MEL-B, or MEL-C.

In this paper, we focused on two kinds of properties in cationic liposomes involved in the early steps of gene transfection. We first investigated the activity of MELs-containing liposomes for DNA capsulation. The high ability in DNA capsulation was considered to lead to the effective delivery of DNA to the surface of target cells. As shown in Fig. 2, the ethidium intercalation assay showed that MEL-A- and MELC-containing liposomes enhanced the DNA capsulation, while MEL-B-containing ones did not. This poor ability in DNA capsulation is probably the reason for its low transfection efficiency of MEL-B-containing liposomes.

Next, we measured the ability in membrane fusion of cationic liposomes with artificial anionic liposomes, because membrane fusion is considered to be an important event to release DNA from complexes. As shown in Fig. 4, we found 
that MEL-A greatly facilitated the level and rate of membrane fusion with anionic liposomes using FRET assay. On the other hand, MEL-B exhibited the similar fusogenic activity as MEL-A, while MEL-C showed the lower activity compared to MEL-A. This lower activity of the membrane fusion is probably the reason for a dim transfection efficiency of MEL-C-containing liposomes.

Thus, the MEL-A-containing liposome has the excellent properties for gene transfection in DNA capsulation and membrane fusion, while MEL-B- and MEL-C-containing liposomes were found to have the poorer properties in DNA capsulation and membrane fusion, respectively. In this study we investigated several physicochemical properties of liposomes containing MELs. At present, we do not have enough explanation why each MEL-containing liposome behaved so differently in physicochemical properties, but elucidation of the relationship between molecular structure of MELs and the difference of properties in MELs-containing liposomes would provide new insights for the discovery of the advanced biosurfactant derivatives. We were able to understand here why the cationic liposomes with MEL-A had the most favorable properties for gene transfection among three kinds of MEL derivatives, suggesting that these properties of MEL-Acontaining liposomes must underlie the high transfection efficiency.

Acknowledgments We thank Dr. D. Kitamoto (National Institute of Advanced Industrial Science and Technology) for supplying MELs.

\section{REFERENCES}

1) Felgner P. L., Gadek T. R., Holm M., Roman R., Chan H. W., Wenz M.,
Northrop J. P., Ringold G. M., Danielsen M., Proc. Natl. Acad. Sci. U.S.A., 84, 7413-7417 (1989).

2) Gao X., Huang L., Biochem. Biophys. Res. Commun., 179, 280-285 (1991).

3) Lasic D. D., J. Control. Release, 48, 203-222 (1997).

4) Khalil I. A., Kogure K., Akita H., Harashima H., Pharmacol. Rev., 58, $32-45$ (2006).

5) Okayama R., Noji M., Nakanishi M., FEBS Lett., 408, 232-234 (1997).

6) Fujiwara C., Hasegawa S., Hirashima N., Nakanishi M., Ohwada T., Biochim. Biophys. Acta, 1468, 396- 402 (2000).

7) Noguchi A., Hirashima N., Furuno T., Nakanishi M., Neurosci. Lett., 325, 29-32 (2002).

8) Noguchi A., Hirashima N., Nakanishi M., Pharm. Res., 19, 933-938 (2002).

9) Kawaura C., Hasegawa S., Hirashima N., Nakanishi M., Biol. Pharm. Bull., 23, 778-780 (2000).

10) Nakanishi M., Curr. Med. Chem., 10, 1289-1296 (2003).

11) Kitamoto D., Akiba S., Hioki T., Tabuchi T., Agric. Biol. Chem., 54, $31-36(1990)$

12) Inoh Y., Kitamoto D., Hirashima N., Nakanishi M., Biochem. Biophys. Res. Commun., 289, 57-61 (2001).

13) Inoh Y., Kitamoto D., Hirashima N., Nakanishi M., J. Control. Release, 94, 423-431 (2004).

14) Noguchi A., Furuno T., Kawaura C., Nakanishi M., FEBS Lett., 433 , $169-173(1998)$

15) Kawaura C., Noguchi A., Furuno T., Nakanishi M., FEBS Lett., 421, 69-72 (1998)

16) Xu Y., Szoka F. C., Biochemistry, 35, 5616-5623 (1996).

17) Sakurai F., Inoue R., Nishino F., Okuda A., Matsumoto O., Taga T., Yamashita F., Takakura Y., Hashida M., J. Control. Release, 66, 255269 (2000).

18) Bailey A. L., Cullis P. R., Biochemistry, 36, 1628-1634 (1997).

19) Struck D. K., Hoekstra D., Pagano R. E., Biochemistry, 20, 4093 4099 (1981).

20) Hasegawa S., Hirashima N., Nakanishi M., Bioorg. Med. Chem. Lett., 12, 1299-1302 (2002) 